\title{
REMOÇÃO DO GLICEROL LIVRE PRESENTE NO BIODIESEL POR SORÇÃO EM VERMICULITA
}

\author{
R. GUIMARÃES ${ }^{1}$, A. E. $\operatorname{COSTA}^{2}$, C. A. K. GOUVÊA ${ }^{3}$ e J. K. ANDREAZZA $^{3}$ \\ ${ }^{1}$ Bolsista de Iniciação Científica - PIBIC/CNPq - Centro Universitário Tupy \\ ${ }^{2}$ Centro Universitário Tupy, Docente do Departamento de Engenharia Química e Orientadora de \\ Iniciação Científica \\ ${ }^{3}$ Centro Universitário Tupy, Docente do Departamento de Engenharia Química \\ E-mail para contato: aelcosta@ hotmail.com
}

\begin{abstract}
RESUMO - Neste trabalho estudou-se a purificação de biodiesel quanto à remoção de glicerol livre utilizando a sorção como um método alternativo à usual lavagem aquosa. Após ser produzido, o biodiesel precisa passar por uma etapa de purificação final visando atender às especificações de qualidade estabelecidas pela ANP. O método da lavagem aquosa possui grandes desvantagens, como o abundante consumo de água e geração de efluentes. Assim, propõe-se a purificação de biodiesel por sorção em vermiculita, material alternativo de baixo custo. Testes cinéticos e de equilíbrio de sorção foram realizados em batelada e temperatura ambiente, sendo realizadas as análises de teor de glicerol livre por cromatografia gasosa. Foram aplicados os modelos de Freundlich e Langmuir para descrição das isotermas. Nos ensaios de equilíbrio, a isoterma de Langmuir representou melhor o comportamento dos materiais, sendo que a vermiculita foi tão eficiente quanto o padrão comercial na remoção de glicerol livre do biodiesel. Dessa maneira, a sorção em vermiculita mostrou-se um método adequado para a purificação de biodiesel, podendo ser empregado em substituição à lavagem aquosa.
\end{abstract}

Palavras-chave: Glicerol Livre, Cinética, Isoterma.

\section{INTRODUÇÃO}

O biodiesel é um combustível derivado de óleos vegetais ou gorduras animais, sendo constituído por ésteres monoalquilícos de ácidos graxos de cadeia longa. O processo usual para sua produção é a reação de transesterificação, onde um álcool de cadeia curta reage com um triglicerídeo na presença de um catalisador (ácido ou básico), formando ésteres monoalquílicos (biodiesel) e glicerol. Destaca-se como alternativa ao diesel por possuir similaridade de propriedades, causar menor impacto ambiental e possibilidade de produção a partir de matérias-primas renováveis (KUCEK, 2004).

Dentre os benefícios da utilização do óleo residual de frituras para produção do biodiesel, podese destacar o seu menor custo, a inadequação para fins alimentícios e o problema constituído por seu 
descarte inadequado. Logo, o seu emprego é vantajoso do ponto de vista econômico e ambiental quando comparado a óleos virgens (COSTA, 2008).

Em 2012 a Agência Nacional do Petróleo, Gás Natural e Biocombustíveis (ANP) publicou a sua Resolução número 14, na qual constam quais devem ser as características que o biodiesel deve apresentar parar ser comercializado (ANP, 2012). A qualidade do biodiesel produzido é um dos fatores que define se a proporção da adição do biodiesel ao óleo diesel irá aumentar ou não, sendo considerada de grande importância para os empresários da área, pois um aumento obrigatório significa maior demanda (FREITAS, 2011).

Após sua produção, o biodiesel precisa passar por uma etapa de purificação final para remoção dos contaminantes. Esta etapa geralmente é realizada pelo método da lavagem aquosa, o qual possui desvantagens como a grande geração de efluentes e a propensão à formação de emulsões (FINEP, 2006). Desse modo, neste trabalho, pretende-se realizar a purificação de biodiesel produzido com metanol e óleo de frituras pelo método da sorção, usando um sorvente alternativo (a vermiculita), de modo a obter um processo de purificação livre da utilização de água, empregando um material de baixo custo. O emprego da etapa de purificação é essencial para que o biodiesel final atenda às especificações da ANP.

Assim, o objetivo desse estudo é desenvolver um método de purificação final de biodiesel proveniente de óleos residuais de fritura por sorção, utilizando vermiculita como sorvente alternativo, visando substituir a tradicional etapa de lavagem aquosa dos ésteres. Por fim, deseja-se avaliar a eficiência do material alternativo em relação à remoção de traços de glicerol livre do biodiesel.

\section{MATERIAIS E MÉTODOS}

\subsection{Biodiesel}

Para realização dos ensaios de purificação utilizou-se biodiesel produzido pela empresa Ecobiosul do Brasil Ltda., localizada na cidade de Araquari - SC. Para sua produção foi utilizado óleo residual de frituras e metanol, submetidos ao processo de transesterificação em batelada. $\mathrm{Na}$ Tabela 1, apresentam-se propriedades do biodiesel usado nos experimentos de sorção.

Tabela 1 - Propriedades iniciais do biodiesel.

\begin{tabular}{|c|c|}
\hline $\begin{array}{c}\text { Densidade }-\boldsymbol{\rho} \\
\left(\mathbf{k g ~ m}^{-\mathbf{3}}\right)\end{array}$ & $\begin{array}{c}\text { Glicerina livre } \mathbf{G L} \\
(\mathbf{\%} \text { massa })\end{array}$ \\
\hline 0,877 & 0,254 \\
\hline
\end{tabular}

\subsection{Sorventes}

Foi selecionado um material com características adsortivas e absortivas para ser empregado como sorvente durante os ensaios, a vermiculita. A fim de comparar os resultados obtidos, utilizou-se 
uma resina comercial específica.

Vermiculita: A vermiculita utilizada nos ensaios custa em média $\mathrm{R} \$ 0,50 / \mathrm{kg}$.

Resina comercial PD206: A resina comercial selecionada é a PD206, fabricada pela Purolite®. Trata-se de uma resina com finalidade específica para purificação de biodiesel obtido pela reação de transesterificação. Seu preço aproximado é de US\$ 14,00/kg, acrescido de 5\% de IPI. A Tabela 2 apresenta algumas características dessa resina.

Tabela 2 - Características da resina PD206 (Purolite®, 2014).

\begin{tabular}{|c|c|}
\hline \multicolumn{2}{|c|}{ Características básicas } \\
\hline Aplicação & Purificação de biodiesel \\
\hline $\begin{array}{c}\text { Estrutura } \\
\text { polimérica }\end{array}$ & $\begin{array}{c}\text { Poliestireno com } \\
\text { divinilbenzeno, tipo gel }\end{array}$ \\
\hline Aparência & Partículas esféricas \\
\hline $\begin{array}{c}\text { Grupo } \\
\text { funcional }\end{array}$ & Ácido sulfônico \\
\hline $\begin{array}{c}\text { Forma iônica } \\
\text { de troca }\end{array}$ & $\mathrm{H}^{+}$ \\
\hline
\end{tabular}

\subsection{Cinéticas de Sorção}

Em trabalho anterior (GUIMARÃES et al., 2014), as cinéticas foram realizadas em batelada à temperatura ambiente e em duplicata. Foram adicionados $40 \mathrm{~mL}$ de biodiesel e $0,5 \mathrm{~g}$ de sorvente em béqueres de $100 \mathrm{~mL}$. Posteriormente, os sistemas foram submetidos à agitação mecânica em tempos pré-determinados entre 1 e 180 minutos. Em seguida, separou-se o sorvente do biodiesel por filtração a vácuo. Por fim, para avaliar a quantidade de glicerol livre nas amostras após o processo de sorção, realizou-se análise de cromatografia gasosa, conforme a norma EN 14105 (CEN, 2010).

\subsection{Isotermas de Sorção}

Procedimento experimental: Os ensaios foram realizados em batelada à temperatura ambiente e em duplicata, semelhante às cinéticas de sorção. Foram adicionados $40 \mathrm{~mL}$ de biodiesel e massa variável de sorvente de $0,1 \mathrm{~g}, 0,2 \mathrm{~g}, 0,3 \mathrm{~g}, 0,4 \mathrm{~g}$ e 0,5 g em béqueres de $100 \mathrm{~mL}$. Logo após, o sistema foi mantido à agitação mecânica por 90 minutos. Novamente utilizou-se filtração a vácuo para remover o sorvente do biodiesel e o método AOCS Ca 56-14 para determinação dos teores de glicerol livre.

Modelos para descrição das isotermas: Foram selecionados dois modelos para analisar o sorvente em estudo, Langmuir e Freundlich.

A isoterma de Langmuir é adequada para adsorção em monocamada em superfícies homogêneas, sendo muito utilizada como ponto de partida para formulações de expressões cinéticas. 
Sua formulação é baseada na hipótese de que as forças de interação entre as moléculas adsorvidas são desprezíveis e que cada sítio é definido e pode ser ocupado por apenas uma molécula (LANGMUIR, 1916).

A expressão da isoterma de Langmuir é apresenta na Equação 1.

$$
q_{e}=\frac{q_{0} \cdot b \cdot C_{e}}{1+b \cdot C_{e}}
$$

A Equação 1 pode ser rearranjada, obtendo-se assim a sua forma linearizada, conforme a Equação 2.

$$
\frac{C_{e}}{q_{e}}=\frac{1}{q_{0} b}+\frac{C_{e}}{q_{0}}
$$

A partir dos parâmetros $\mathrm{C}_{0} \mathrm{e} \mathrm{b}$, podemos prever as características essenciais da isoterma de Langmuir utilizando o fator adimensional de separação RL, apresentado pela Equação 3. O valor de RL indica o tipo de isoterma de adsorção obtido, além de fornecer informações a respeito do equilíbrio de adsorção envolvido, na Tabela 3 apresenta-se esse parâmetro.

$$
R L=\frac{1}{1+\mathrm{C}_{0} \mathrm{~b}}
$$

Tabela 3 - Tipo de isoterma de acordo com o valor do fator de separação RL.

\begin{tabular}{|c|c|}
\hline RL & Tipo de isoterma \\
\hline RL $>1$ & $\begin{array}{c}\text { Equilíbrio } \\
\text { Desfavorável }\end{array}$ \\
\hline $\mathrm{RL}=1$ & Caso Linear \\
\hline $0<\mathrm{RL}<1$ & Equilíbrio Favorável \\
\hline $\mathrm{RL} \rightarrow 0$ & Caso Irreversível \\
\hline
\end{tabular}

O modelo de Freundlich, apresentado na Equação 4 também é muito utilizado na descrição das isotermas, é apropriado para representar as superfícies heterogêneas, além de considerar a existência de multicamadas adsorvidas. Seu emprego também sugere que a energia de sorção decresce exponencialmente com a ocupação dos sítios ativos do adsorvente (FREUNDLICH, 1906; CRINI et $a l .$, 2007). Esse modelo é a mais antiga equação para descrever o processo de sorção.

$$
q_{e}=K_{F} C_{e}{ }^{n}
$$

A Equação 4 pode ser rearranjada, obtendo-se a forma linearizada do modelo de Freundlich, conforme a Equação 5. 
$\log q_{e}=\log K_{F}+n \log C_{e}$

O modelo apresenta melhores resultados para casos em que apenas um soluto está presente no meio. No entanto, a desvantagem desta isoterma empírica é que para concentrações de soluto próximas a zero, o modelo não se reduz à Lei de Henry, o que limita sua faixa de concentração, segundo Fritz e Schlünder (1981).

\section{RESULTADOS E DISCUSSÃO}

\subsection{Cinéticas de Sorção}

Na Figura 1 são apresentados os resultados cinéticos, obtidos em trabalho anterior (Guimarães et al., 2014), referentes ao teor de glicerol livre versus tempo para os materiais analisados.

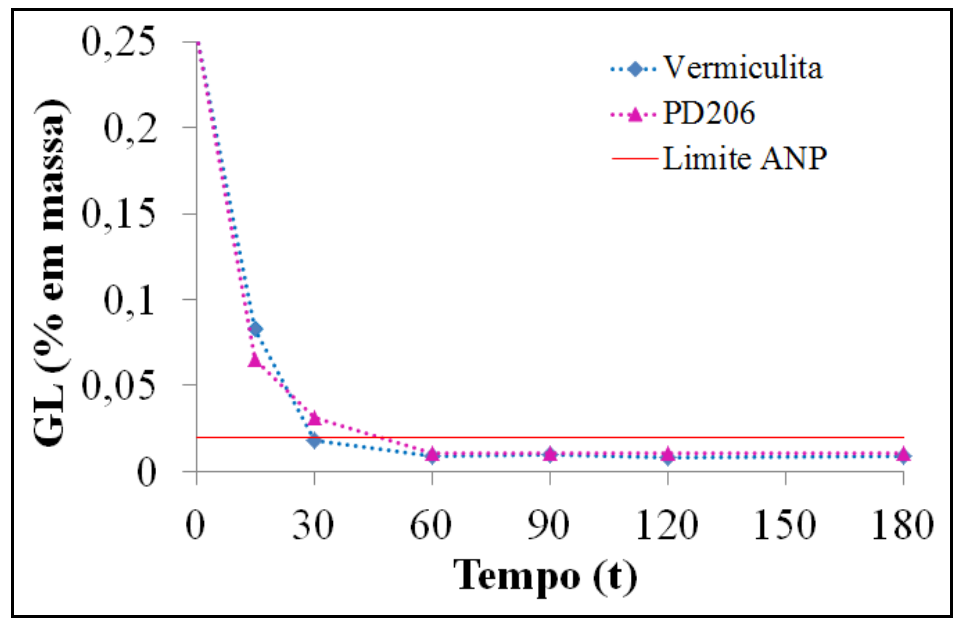

Figura 1 - Cinéticas de adsorção do glicerol livre do biodiesel em vermiculita e em PD206.

A partir da Figura 1, observa-se que a vermiculita e a resina comercial alcançaram índices menores do que o valor máximo de $0,02 \%$ em massa de glicerol livre estipulado pela ANP. Porém, a vermiculita reduziu os teores mais rapidamente, em cerca de 30 minutos. A resina comercial levou cerca de 60 minutos para alcançar o valor estabelecido pela especificação da ANP.

\subsection{Isotermas de Sorção}

As Figuras 3 e 4 foram obtidas a partir da aplicação do modelo de Langmuir linearizado (Equação 2) e representam as isotermas de sorção do glicerol livre em vermiculita e em resina PD206. 


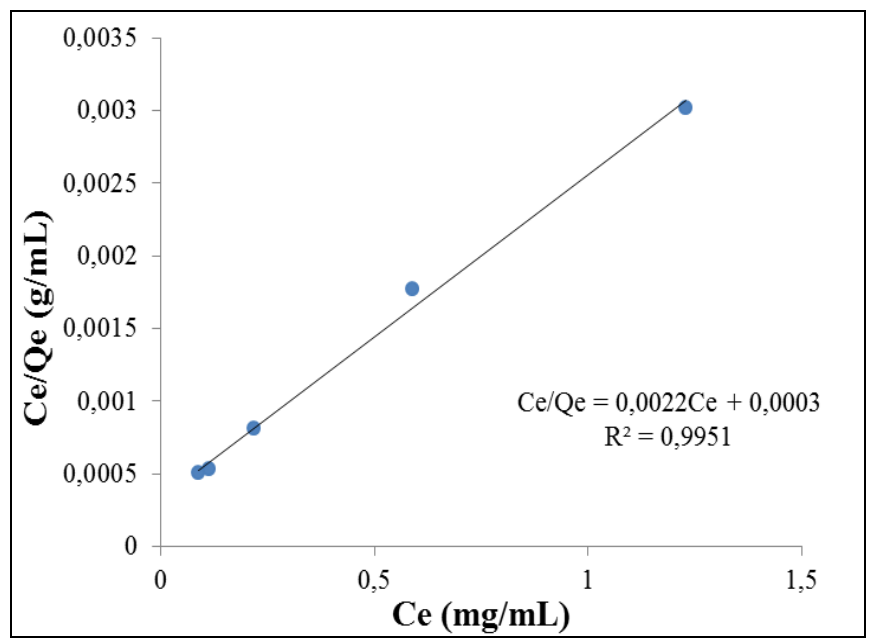

Figura 3 - Isoterma de sorção de glicerol livre do biodiesel em vermiculita.

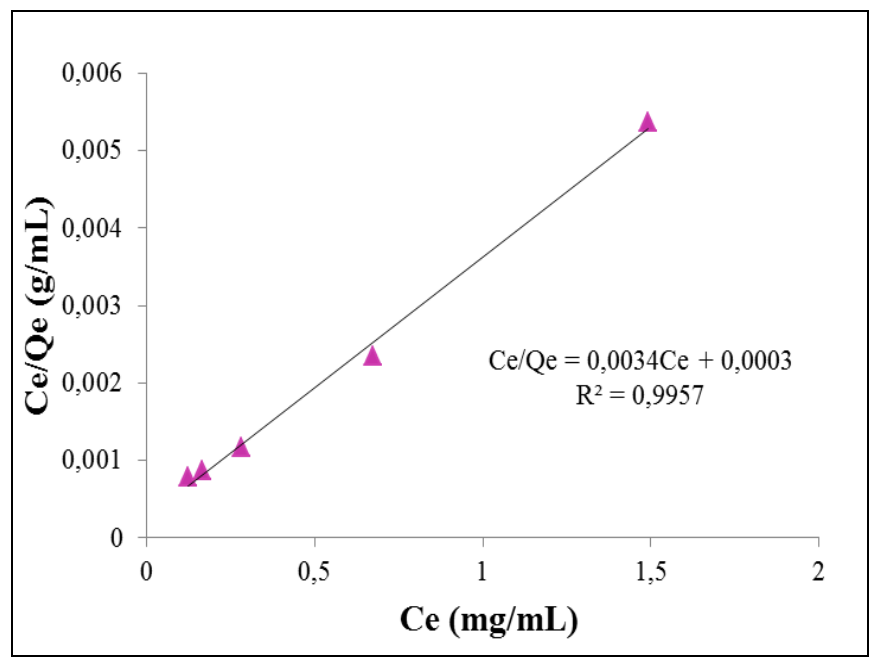

Figura 4 - Isoterma de sorção de glicerol livre do biodiesel em PD206.

Na Tabela 4 são apresentados os coeficientes obtidos com a aplicação da isoterma de Langmuir aos dados experimentais. Os coeficientes de regressão linear $\left(\mathrm{R}^{2}\right)$ apresentados na Tabela 4 indicam que os dados experimentais ajustaram-se bem ao modelo de Langmuir, para ambos os sorventes. Já pelo coeficiente $\mathrm{q}_{0}$ pode-se observar a maior capacidade de sorção de glicerol livre por parte da vermiculita em relação ao valor da resina PD206. Com relação ao parâmetro RL, os valores abaixo de 1 indicam que o equilíbrio de adsorção é favorável, ressaltando a viabilidade do método.

Tabela 4 - Coeficientes do modelo de Langmuir para isotermas de sorção.

\begin{tabular}{|c|c|c|c|c|}
\hline Sorvente & $\mathbf{q}_{\mathbf{0}}(\mathbf{m g} \mathbf{g})$ & $\mathbf{b} \mathbf{( m L / m g})$ & $\mathbf{R L}$ & $\mathbf{R}^{\mathbf{2}}$ \\
\hline Vermiculita & 454,5 & 7,3 & 0,98 & 0,9951 \\
\hline PD 206 & 294,1 & 11,3 & 0,97 & 0,9957 \\
\hline
\end{tabular}


O modelo de Freundlich, no entanto, não obteve o mesmo sucesso na representação dos dados em estudo. Além disso, a presença de cálculos logarítmicos em pontos com concentrações de glicerol livre iguais a zero deram origem a indeterminações. Dessa forma, este modelo não mostrou-se adequado para avaliação do comportamento dos processos de sorção em estudo.

\section{CONCLUSÕES}

Com base nos resultados dos ensaios cinéticos de sorção pode-se concluir que tanto a vermiculita quanto a diatomita apresentaram bom desempenho na remoção de glicerol livre do biodiesel, apresentando comportamentos similares ao da resina comercial específica para essa finalidade. $\mathrm{O}$ uso de ambos os sorventes resultou em biodiesel com teor de glicerol livre permitido pela ANP (0,02\% em massa), em um tempo de sorção de 30 minutos.

Sobre os modelos para descrição das isotermas, o modelo de Langmuir representou satisfatoriamente os dados experimentais. Através de seus parâmetros foi possível avaliar que a vermiculita apresentou comportamento de equilíbrio similar ao da resina comercial PD206.

Os resultados apresentados demonstram que a sorção é um método viável para substituir a usual lavagem aquosa na purificação do biodiesel e que a vermiculita mostra-se como sorvente eficiente na remoção de traços de glicerol livre do biocombustível.

\section{NOMENCLATURA}

b: Parâmetro relacionado à afinidade (mL líquido/mg sólido)

C: Constante que representa o coeficiente linear do modelo de difusão intraparticular (mg sólido/g sorvente)

$\mathrm{C}_{\mathrm{e}}$ : Concentração de equilíbrio do adsorbato na fase líquida(mg sólido/ $\mathrm{mL}$ líquido)

$\mathrm{C}_{0}$ : Concentração inicial de GL do biodiesel $[\mathrm{g} / \mathrm{mL}]$

$\mathrm{K}_{\mathrm{F}}$ : Constante de Freundlich

ki: Constante de difusão intraparticular ( $\mathrm{mg}$ sólido/g sorvente $\min ^{1 / 2}$ )

$\mathrm{k}_{1}$ : Constante da equação cinética de pseudo-primeira ordem $\left(\mathrm{min}^{-1}\right)$

$\mathrm{k}_{2}$ : Constante da equação cinética de pseudo-segunda ordem (g sorvente/mg sólido min)

$\mathrm{q}_{\mathrm{e}}$ : Quantidade de sólido presente no sorvente no equilíbrio (mg sólido/g sorvente)

$\mathrm{q}_{\mathrm{t}}$ : Quantidade de sólido presente no sorvente no tempo $\mathrm{t}$ ( $\mathrm{mg}$ sólido/g sorvente)

$\mathrm{q}_{0}$ : Massa máxima de soluto adsorvido na monocamada (mg sólido/ g adsorvente)

n: Coeficiente empírico do modelo de Freundlich

RL: Fator adimensional de separação

t: Tempo (min)

\section{REFERÊNCIAS BIBLIOGRÁFICAS}

ANP - AGÊNCIA NACIONAL DO PETRÓLEO, GÁS NATURAL E BIOCOMBUSTÍVEL. Resolução ANP $\mathrm{N}^{\circ} 14$ de 2012. Disponível em: < http://nxt.anp.gov.br/nxt/gateway.dll/leg/ 


\section{9 a 22 de outubro de 2014 \\ Florianópolis/SC}

resolucoes_anp/2012/maio/ranp\%2014\%20-\%202012.xml >. Acesso em: 30 abr. 2014.

CEN - COMITÉ EUROPÉEN DE NORMALISATION. EN 14214 - Automotive fuels - Fatty acid methyl esters (FAME) for diesel engines - Requirements and test methods, 2010.

COSTA, A.E. Purificação por adsorção de biodiesel etílico obtido por transesterificação de óleo residual de fritura. 2008, 162p. Tese (Qualificação para Doutorado em Engenharia Química) Universidade Federal de Santa Catarina, UFSC-SC, Florianópolis.

CRINI, G.; PEINDY, H.N.; GIMBERT, F.; ROBERT, C. Removal of C.I. basic green 4 (Malachite Green) from aqueous solutions by adsorption cyclodextrin-based adsorbent: Kinetic and equilibrium studies. 2007, Elsevier, v. 57, p. 97-110.

FINANCIADORA DE ESTUDOS E PROJETOS - FINEP. Chamada pública MCT/FINEP, Ação Transversal Biodiesel 10/2006. Disponível em: <http://www.finep.gov.br// fundos_setoriais/acao_transversal/editais/Chamada_Publica_MCT_FINEP_Acao_Transversal_Biodie sel_10_2006.pdf> Acesso em: março de 2007.

FREITAS, R.C.; DUARTE, A. Controle de qualidade. BiodieselBR, Curitiba: BiodieselBR, n.21, fev./mar. $2011.58 \mathrm{p}$.

FREUNDLICH, H. M. F. Über die adsorption in lösungen. Zeitschrift für Physikalische Chemie, v. 57, p. 385-470, 1906.

FRITZ W.; SCHLÜNDER, E. U. Competitive adsorption of two dissolved organics onto activated carbon-I: Adsorption equilibria. Chemical Engineering Science, v. 36, n. 4, p 731-741, 1981.

GUIMARÃES, R. et al. Purificação de biodiesel produzido com óleo de frituras por sorção em vermiculita. X Congresso Brasileiro de Engenharia Química Iniciação Científica, 2014.

KUCEK, K. T., 2004. Otimização da transesterificação etílica do óleo de soja em meio alcalino, Universidade Federal do Paraná, Curitiba-PR. (Dissertação de Mestrado em Química Orgânica).

LANGMUIR, I. The constitution and fundamental properties of solids and liquids. Journal of the American Chemical Society, v. 38, p. 2221-2295, 1916.

PUROLITE®. PUROLITE® PD206, 2014. Disponível em: <http://sa.purolite.com/RelId/612322/ ISvars/default/Purolite_PD206.htm>. Acesso em: 30 abr. 2014.

\section{AGRADECIMENTOS}

Ao Conselho Nacional de Desenvolvimento Científico e Tecnológico (CNPq) e ao Centro Universitário Tupy (UNISOCIESC). Às empresas Purolite ${ }^{\circledR}$ e Ecobiosul do Brasil Ltda. pelo fornecimento de amostras de adsorvente e biodiesel, respectivamente. 